\title{
A new era in cervical cancer prevention
}

\section{Lara Roeske}

There are no old roads to new directions.

- Boston Consulting Group

A NEW ERA in cervical cancer prevention is here. For general practitioners (GPs), practice teams and patients, this heralds a time of change and challenge. I have noted the transformation of cervical screening at my practice and at the practices of many GP colleagues in response to the commencement of Australia's renewed National Cervical Screening Program (NCSP) on 1 December 2017. GPs are required to update approaches to cervical screening, including informing practice staff and updating practice systems to incorporate key components of the program into clinical practice. The renewed approach to cervical screening involves testing for the presence of the causal agent for cervical cancer, human papillomavirus (HPV).

The NCSP is a program based on current evidence and best practice, and is for all women aged 25-74 years who have ever been sexually active, regardless of whether they have been vaccinated against HPV. For the first time, GPs can include HPV-based testing for the investigation of women with abnormal vaginal bleeding and other possible symptoms of cervical cancer. Additionally, GPs can access the self-collection pathway for eligible underscreened and never-screened women to enable these women to participate in cervical screening. The National Guidelines, ${ }^{1}$ endorsed by The Royal Australian College of General Practitioners, have been developed following a comprehensive review of cervical cancer screening evidence ${ }^{2}$ and will assist GPs to implement the renewed program.

GPs are critical to the successful implementation of the renewed NCSP, having contributed to the remarkable success of the previous program. ${ }^{3,4}$ Moreover, GPs are at the frontline of educating women on the safety of the program and the potential benefits of participation in cervical screening, and supporting the transition of women from the previous program to the new program. GP education and confidence in the new program are therefore paramount for its successful implementation. GPs can be confident that a five-yearly HPV test is a safe and more effective primary screening test that affords increased protection against the development of cervical cancer when compared with twoyearly Pap smears. ${ }^{5}$ Furthermore, HPV tests used in the program are calibrated to detect HPV at levels predictive of the presence of underlying cervical intraepithelial neoplasia rather than any HPV at all. ${ }^{6}$

Despite the success of the previous program based on two-yearly Pap smears, there has been no change in the incidence and mortality associated with adenocarcinoma of the cervix. The renewed program, based on five-yearly HPV testing, is predicted to deliver an additional decline of $24-36 \%$ in the incidence and mortality from cervical cancer by enhancing the rate of detection of precursors of adenocarcinoma and squamous cell cervical cancers. ${ }^{7,8}$ This is reassuring for GPs and their patients. Australian women will be the beneficiaries of a less frequent, safe and more effective cervical screening program.

Conceivably, the scale of the evidencebased changes to cervical screening in general practice ushered in by the renewed NCSP could be a milestone towards the eradication of cervical cancer in Australia. Thus, for current and future generations of Australian women, the renewed HPV-based NCSP, along with HPV vaccination, will deliver even greater protection against cervical cancer than ever before. $^{9}$

\begin{abstract}
Author
Lara Roeske BMedSc, MBBS (Hons), FRACGP, is a Melbourne general practitioner and practice owner director of Education and Liaison at the Victorian Cytology Service; chair of the RACGP Specific Interests Sexual Health Medicine Network; member of the Cancer Council Australia Cervical Cancer Screening Guidelines Working Party; and coauthor of the National Cervical Screening Program: Guidelines for the management of screen-detected abnormalities, screening in specific populations and investigation of abnormal vaginal bleeding.
\end{abstract}

\section{References}

1. Cancer Council Australia. Cervical cancer screening: National Cervical Screening Program: Guidelines for the management of screendetected abnormalities, screening in specific populations and investigation of abnormal vaginal bleeding. Sydney: Cancer Council Australia, 2016. Available at http://wiki.cancer.org.au/australia/ Guidelines:Cervical_cancer/Screening [Accessed 1 December 2017].

2. Medical Services Advisory Committee. Application no. 1276 - Renewal of the National Cervical Screening Program (public summary document). Canberra: Commonwealth of Australia, 2014 Available at http://msac.gov.au/internet/msac/ publishing.nsf/Content/1276-public [Accessed 1 January 2018]

3. Australian Institute of Health and Welfare. General practice activity in Australia 2009-10. Cat no. GEP2. Canberra: AIHW, 2010. Available at www. aihw.gov.au/publication-detail/?id=6442472433 [Accessed 1 January 2018].

4. Munro A, Pavicic $H$, Leung $Y$, et al. The role of general practitioners in the continued success of the National Cervical Screening Program. Aust Fam Physician 2014;43(5):293-96.

5. Dilner J, Rebolj M, Birembaut P, et al. Long term predictive values of cytology and human papillomavirus testing in cervical cancer screening: Joint European cohort study. BMJ 2008;337:a1754. doi: 10.1136/bmj.a1754.

6. Hawkes D. Human papillomavirus testing as part of the renewed National Cervical Screening Program. Aust J Gen Pract 2018;47(7):412-14.

7. Ronco G, Dillner J, Elfström KM, et al. Efficacy of HPV-based screening for prevention of invasive cervical cancer: Follow-up of four European randomised controlled trials. Lancet 2014;383(9916):524-32. doi: 10.1016/S01406736(13)62218-7.

8. Lew JB, Simms KT, Smith MA, et al. Primary HPV testing versus cytology-based cervical screening in women in Australia vaccinated for HPV and unvaccinated: Effectiveness and economic assessment for the National Cervical Screening Program. Lancet Public Health 2017;2(2):96-107. doi: 10.1016/S2468-2667(17)30007-5.

9. Brotherton J. Human papillomavirus vaccination update: Nonavalent vaccine and the two-dose schedule. Aust J Gen Pract 2018;47(7):417-21. 